\title{
Voluntary Control of Human Jaw Stiffness
}

\author{
Douglas M. Shiller, ${ }^{1}$ Guillaume Houle, ${ }^{1}$ and David J. Ostry ${ }^{1,2}$ \\ ${ }^{1}$ Department of Psychology, McGill University, Montreal, Quebec, Canada; and ${ }^{2}$ Haskins Laboratories, New Haven, Connecticut
}

Submitted 15 February 2005; accepted in final form 14 June 2005

Shiller, Douglas M., Guillaume Houle, and David J. Ostry. Voluntary control of human jaw stiffness. J Neurophysiol 94: 2207-2217, 2005. First published June 22, 2005; 10.1152/jn.00164.2005. Recent studies of human arm movement have suggested that the control of stiffness may be important both for maintaining stability and for achieving differences in movement accuracy. In the present study, we have examined the voluntary control of postural stiffness in 3D in the human jaw. The goal is to address the possible role of stiffness control in both stabilizing the jaw and in achieving the differential precision requirements of speech sounds. We previously showed that patterns of kinematic variability in speech are systematically related to the stiffness of the jaw. If the nervous system uses stiffness control as a means to regulate kinematic variation in speech, it should also be possible to show that subjects can voluntarily modify jaw stiffness. Using a robotic device, a series of force pulses was applied to the jaw to elicit changes in stiffness to resist displacement. Three orthogonal directions and three magnitudes of forces were tested. In all conditions, subjects increased the magnitude of jaw stiffness to resist the effects of the applied forces. Apart from the horizontal direction, greater increases in stiffness were observed when larger forces were applied. Moreover, subjects differentially increased jaw stiffness along a vertical axis to counteract disturbances in this direction. The observed changes in the magnitude of stiffness in different directions suggest an ability to control the pattern of stiffness of the jaw. The results are interpreted as evidence that jaw stiffness can be adjusted voluntarily, and thus may play a role in stabilizing the jaw and in controlling movement variation in the orofacial system.

\section{N T R O D U C T I O N}

The need for precise control of jaw position and force is central to orofacial behaviors such as speech and mastication. Speech movements in particular require a high degree of precision and indeed the notion that speech sounds differ in terms of their precision requirements is a key idea in speech production research (Beckman et al. 1995; Gay et al. 1992; Perkell and Nelson 1985; Stevens 1989). There is some evidence that the movement of different articulators may be coordinated in a reciprocal fashion to constrain acoustical variation (Guenther et al. 1999; Perkell et al. 1993; Savariaux et al. 1995). Here we assess another possibility, that differences in kinematic precision may be achieved through centrally controlled modifications to articulator stiffness.

The present paper focuses on the control of human jaw stiffness and in particular on two possible functions of stiffness control: maintaining position and controlling variation. In work to date on human arm movement, it has been shown that stiffness - the change in resistive force that results from a change in position-is substantially dependent on limb geometry and counteracts the effects of external loads (Flash and

Address for reprint requests and other correspondence: D. J. Ostry, Department of Psychology, McGill University, 1205 Dr. Penfield Ave., Montreal, QC, Canada H3A 1B1 (E-mail: ostry@motion.psych.mcgill.ca).
Mussa-Ivaldi 1990; Milner 2002). Evidence that changes in stiffness act to offset potential instabilities has been obtained under static conditions (Darainy et al. 2004; Gomi and Osu 1998; McIntyre et al. 1996; Perreault et al. 2002) as well as during movement (Burdet et al. 2001; Franklin et al. 2003). In multi-degree-of-freedom systems (including the jaw) there is the added possibility of changing the spatial pattern of stiffness. The idea that stiffness is linked to variability is supported by studies of human arm movement in which variability in endpoint position has been related to cocontraction both during movement and at movement end (Gribble et al. 2003). Evidence that stiffness may be linked to variability also comes from studies of jaw movement in speech in which it has been shown that variability in vowel production is related to articulator stiffness (Shiller et al. 2002).

If stiffness control is to play a role in modifying variability in speech, it should be possible to show that patterns of stiffness can be modified in a purposive manner. Here, we have examined the voluntary control of stiffness in three dimensions (3D) by applying single-axis disturbances to the jaw using a robotic device. We have tested the idea that stiffness may be scaled in magnitude in an adaptive fashion to counteract the effects of a disturbance. We have also examined the extent to which the spatial pattern of stiffness may be selectively modified so as to counteract mechanical disturbances that act in different directions. The results support the idea that stiffness magnitudeand in some cases the spatial distribution of stiffness - can be controlled in the jaw, and thus may play a role in stabilizing the jaw and in controlling variability during speech production.

\section{METHODS}

Seven subjects were tested (three females and four males, ages 24 to $29 \mathrm{yr}$ ), none of whom reported any history of temporomandibular joint dysfunction or speech motor disorder.

Jaw stiffness was measured using a robotic device (Phantom Premium 1.0, Sensable Technologies, Woburn, MA) that was operated under open-loop force control and was capable of delivering forces and measuring positions in $3 \mathrm{D}$ with a position resolution of $0.03 \mathrm{~mm}$. The robot was coupled to the jaw using a custom-molded acrylic and metal dental appliance that was attached to the buccal surface of the mandibular teeth using a dental adhesive. A magnesium and titanium rotary connector allowed for jaw motion in all six rotational and translational degrees of freedom and enabled forces applied by the robot to displace the jaw in the three spatial dimensions tested. Subject-applied forces were measured using a six-axis force/ torque sensor mounted on the tip of the robot. Jaw position and force were sampled at $1,000 \mathrm{~Hz}$ (for further details see Shiller et al. 2002).

The initial phase of the experiment (the null condition) involved the estimation of jaw stiffness in the absence of any other manipulation.

\footnotetext{
The costs of publication of this article were defrayed in part by the payment of page charges. The article must therefore be hereby marked "advertisement" in accordance with 18 U.S.C. Section 1734 solely to indicate this fact.
} 
Subjects were instructed to maintain a static jaw position while a series of small ramp-and-hold force perturbations (1 N, 50-ms rise time, 250-ms hold time) was applied. The maintained jaw position corresponded to the production of the midvowel $e$ (as in "fed"). This position was chosen because it was produced near the middle of the jaw's workspace and was comfortable to maintain over an extended period of time. The perturbations were delivered in 18 directions spaced equally about a sphere. The order of delivery was randomized and a 1-s pause occurred between each perturbation. The series of 18 perturbations was applied twice, resulting in a total of 36 perturbations.

To facilitate the maintenance of jaw position during this task, visual feedback of position was provided on a computer screen located in front of the subject. Before the perturbation sequence, the subject produced a sustained vowel for several seconds at which point the position of the jaw (taken at the mandibular central incisors) was measured and displayed. The subject was instructed to keep the jaw as close as possible to the displayed target position without any further vocalization. Jaw perturbations were delivered only if the jaw was held stationary (tangential velocity $<10 \mathrm{~mm} / \mathrm{s}$ for $250 \mathrm{~ms}$ ) at a distance of $<0.75 \mathrm{~mm}$ from the desired position. During the delivery of a perturbation, visual feedback of jaw position was not provided. Subjects were instructed to maintain the jaw stationary during the sequence of perturbations and not to intervene voluntarily.

After the measurement of stiffness in the null condition, a series of trials was carried out using a task that was designed to elicit an increase in jaw stiffness. The task involved the rapid application of force pulses (100-ms duration) during which subjects were required to maintain a stationary jaw position, that is, to counteract the effects of the external disturbance. Specifically, the jaw had to be maintained within $\pm 0.75 \mathrm{~mm}$ of a target position corresponding to the production of the vowel $e$ (maintained without voicing).

The force pulses were applied along a single axis (see following text for details) at a rate of $5 \mathrm{~Hz}$. The forces were not applied cyclically, but rather in a randomized sequence that rendered the direction of force application unpredictable. The random sequence of forces was applied for a period of 2-4 s. The precise duration of the sequence was determined by the subject's performance. If, after $2 \mathrm{~s}$ of disturbance input, the subject had been able to maintain a stationary jaw position for the final $500 \mathrm{~ms}$, a single ramp-and-hold perturbation that permitted the measurement of jaw stiffness was delivered. If subjects were unable to stabilize the jaw within $4 \mathrm{~s}$, the force pulses were stopped and a 5-s "rest" period was provided before restarting the trial. These steps were repeated until perturbations had been delivered in all directions used in the estimation of stiffness.

The procedure for stiffness estimation was exactly the same as that used in the null condition. To ensure that the jaw was initially stationary for this measurement, a $100-\mathrm{ms}$ period of zero force separated the application of the force pulses and the ramp-and-hold perturbation for estimating stiffness. During the application of the force pulses, feedback of jaw position was provided using the visual display described above. However, as in the null condition, no visual feedback was provided during the application of the ramp-and-hold perturbation that was used to estimate stiffness.

Nine conditions were tested, involving three force magnitudes (ranging on an individual basis from 0.57 to $1.19 \mathrm{~N}$ ) and three orthogonal directions of force application (see following text for details). For each combination of the three directions and three magnitudes, jaw stiffness was quantified by a set of 36 ramp-and-hold perturbations $(2 \times 18$ perturbation directions $)$. For all subjects, the three force levels were presented in increasing order. The three different force directions were tested on different days with the order of directions randomized across subjects.

\section{Data analysis}

Measures of jaw position and force were initially represented in a head-centered coordinate system with an origin at the tip of the maxillary central incisors. The $Z$-axis of this coordinate system was aligned with the occlusal axis in the midsagittal plane (the negative direction corresponds to jaw protrusion). The $Y$-axis was roughly vertical and was orthogonal to the $Z$-axis in the sagittal plane (jaw lowering is in the negative direction). The $X$-axis was a lateral axis and was orthogonal to the sagittal plane.

Sample data from an individual trial are shown in Fig. 1. The data are for a single subject in a condition involving forces applied along the lateral $(X)$ axis. Figure $1 A$ shows measured jaw position and restoring force for a random sequence of force pulses $(0.7 \mathrm{~N}$ in magnitude) followed by the ramp-and-hold perturbation used to estimate stiffness. Note that although there is some jaw movement associated with the applied force pulses, it is within the tolerance of the task $( \pm 0.75 \mathrm{~mm})$ and is substantially reduced in comparison with the displacement observed for a $0.7-\mathrm{N}$ load applied under null conditions. Figure $1 B$ shows the perturbation used for stiffness estimation in greater detail.

As the ramp-and-hold perturbation is delivered (Fig. $1 B$ ), the jaw is displaced from its prior rest position. With the commanded force held constant, the jaw maintains a new static position. For purposes of data analysis, measures of jaw position and restoring force were averaged over two time windows, one immediately before the perturbation (20 $\mathrm{ms}$ in duration, labeled W1) and one during the "hold" phase $(50 \mathrm{~ms}$ in duration, beginning $80 \mathrm{~ms}$ after the onset of the perturbation, labeled W2). The difference between the two position values provides a measure of jaw displacement. The difference between the two forces gives a measure of restoring force related to the perturbation. The displacement and associated restoring force together provide a measure of the jaw's springlike behavior or stiffness. Because the jaw is essentially stationary at the displaced position, the contribution of viscosity and inertia may be neglected.

The example shown in Fig. $1 B$ is typical in that there is no voluntary movement in response to the perturbation. In general, jaw motion during the hold phase of the perturbation was negligible. In cases where the range of jaw position within either of the two analysis windows (W1 and W2) exceeded $0.1 \mathrm{~mm}$ in any degree of freedom, the data from that perturbation were excluded from further processing. Such occurrences were infrequent, occurring in $<5 \%$ of all trials.

Jaw stiffness in $3 \mathrm{D}$ is given by the following equations

$$
\begin{aligned}
& \mathrm{d} F_{x}=-K_{x x} \mathrm{~d} x-K_{x y} \mathrm{~d} y-K_{x z} \mathrm{~d} z \\
& \mathrm{~d} F_{y}=-K_{y x} \mathrm{~d} x-K_{y y} \mathrm{~d} y-K_{y z} \mathrm{~d} z \\
& \mathrm{~d} F_{z}=-K_{z x} \mathrm{~d} x-K_{z y} \mathrm{~d} y-K_{z z} \mathrm{~d} z
\end{aligned}
$$

where $\mathrm{d} F_{x}, \mathrm{~d} F_{y}$, and $\mathrm{d} F_{z}$ correspond to lateral, vertical, and horizontal restoring force; $\mathrm{d} x, \mathrm{~d} y$, and $\mathrm{d} z$ are displacements; and $K$ is stiffness measured at the incisors. The individual stiffness values relate restoring force to displacement. Thus for example, $K_{x y}$ relates restoring force in $x$ to displacement in $y$ and so forth.

For each of the three directions of applied force and three force magnitudes, the values of stiffness were obtained by least-squares regression using the mean forces and mean displacements associated with the 36 perturbations. An initial estimate of stiffness under null conditions was also obtained using this procedure.

In previous studies characterizing planar (two-dimensional [2D]) stiffness in the limb and jaw, the values of the $2 \times 2$ stiffness matrix have been represented graphically as an ellipse (Mussa-Ivaldi et al. 1985; Shiller et al. 2002) in which the major axis corresponds to the direction and magnitude of maximum stiffness and the minor axis corresponds to the direction and magnitude of least stiffness. Jaw stiffness in 2D is anisotropic, with a major axis in the direction of jaw protrusion and retraction and a minor axis in the direction of jaw raising and lowering (Shiller et al. 2002). In the present study, the concept of the stiffness ellipse is extended to allow for the graphical depiction of a $3 \times 3$ stiffness matrix. The result is an ellipsoid that characterizes jaw stiffness in $3 \mathrm{D}$. 
A
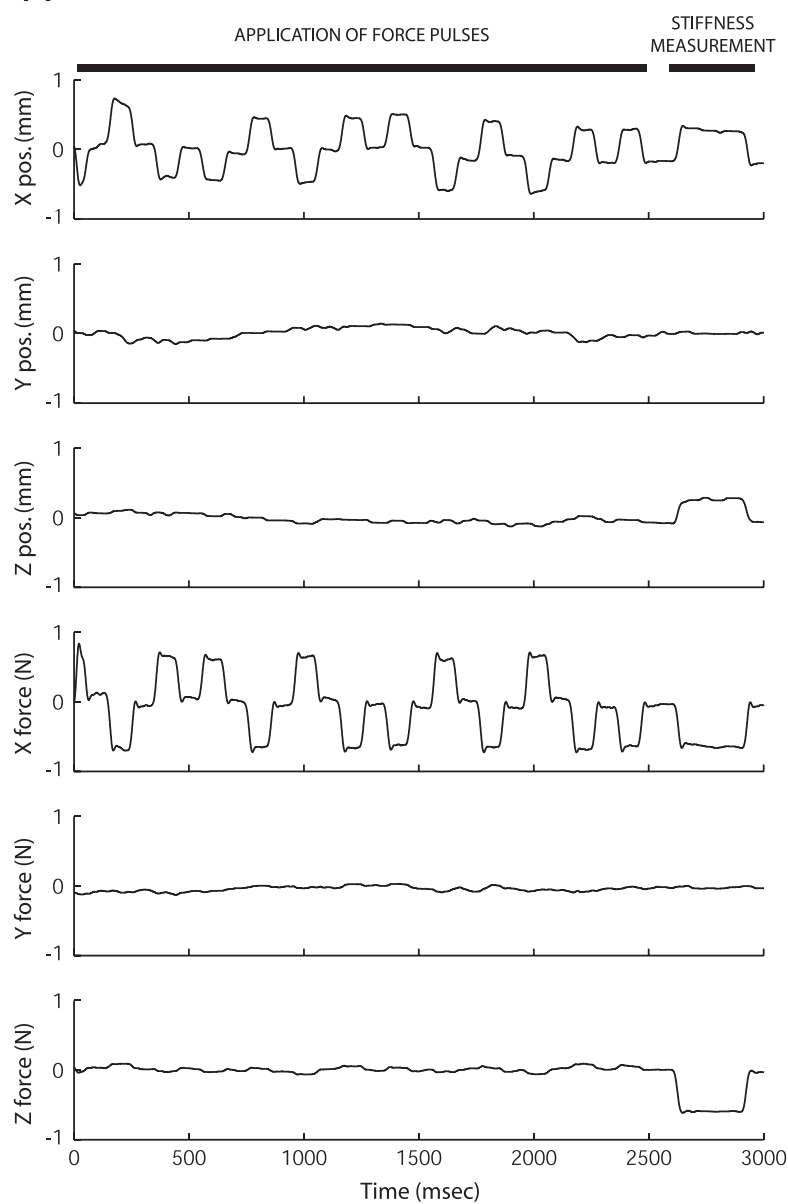

The terms in the stiffness matrix are related to geometrical properties of the ellipsoid. The sign of the off-diagonal terms in the matrix gives the orientation of the ellipsoid relative to the coordinate axes (clockwise vs. anticlockwise); the magnitude of the off-diagonal terms determines the amount of rotation. When the off-diagonal terms are zero the ellipsoid is aligned with the coordinate axes such that the major axis corresponds to the largest term on the diagonal. The symmetry of the off-diagonal terms gives a measure of the springlike behavior of the system. When the off-diagonal terms are equal, restoring forces act in a direction that is opposite to the displacement. When off-diagonal terms are unequal, displacements in $x$ result in restoring forces in $y$ (as an example). The matrices for the null condition given in Table 1 have off-diagonal terms that are both negative and positive and relatively small in magnitude relative to the terms on the diagonal. This combination results in ellipses that are rotated down from the horizontal and very slightly out of the sagittal plane (see following text).

Figure $2 A$ (left) shows a representative ellipsoid under no load conditions. The ellipsoid is characteristic of others in this data set in that it is essentially symmetrical about the midsagittal plane. For purposes of reporting values across subjects, we use a projection of the ellipsoid onto the midsagittal plane and a lateral axis that is perpendicular to the midsagittal plane (Fig. 2A, right). The convention corresponds to the axes defined by the disturbance inputs (see following text). The primary characteristics of stiffness in 3D are reflected in the projection of the 3D ellipsoid onto the midsagittal plane. For example, the major axis of the stiffness ellipsoid was on average $1.33^{\circ}$ from the midsagittal plane. The magnitude of the major axis of the stiffness ellipsoid was almost identical to the magnitude of the projection of the major axis onto the midsagittal plane (the difference
FIG. 1. Sample data from an individual trial involving forces ( $0.7 \mathrm{~N}$ magnitude) applied along the lateral axis. A: measured jaw position and restoring force for a sequence of force pulses followed by the ramp-and-hold perturbation used to estimate stiffness. $B$ : detail of the perturbation used for stiffness estimation. 
TABLE 1. Values for jaw stiffness under null conditions

Estimated Jaw Stiffness Matrix

$$
\left[\begin{array}{ccc}
K_{x x} & K_{x y} & K_{x z} \\
K_{y x} & K_{y y} & K_{y z} \\
K_{z x} & K_{z y} & K_{z z}
\end{array}\right]
$$

\begin{tabular}{crrr}
\hline Subject & & \multicolumn{2}{c}{ Values } \\
\hline \multirow{2}{*}{1} & -0.6988 & 0.0182 & 0.0178 \\
& 0.0109 & -1.0830 & 0.0849 \\
& -0.0523 & -0.1702 & -1.5434 \\
2 & -0.8849 & 0.0857 & 0.1005 \\
& 0.0143 & -1.0155 & -0.0705 \\
& -0.0122 & -0.3185 & -1.4356 \\
3 & -0.6810 & 0.0127 & 0.0552 \\
& 0.0001 & -1.0624 & -0.0946 \\
& -0.0902 & -0.3022 & -1.1597 \\
4 & -0.7416 & 0.0370 & 0.0579 \\
& 0.0041 & -0.9322 & -0.2832 \\
& -0.0164 & -0.4417 & -1.2817 \\
5 & -0.9997 & 0.0205 & 0.0484 \\
& 0.0165 & -1.1262 & -0.1103 \\
& -0.0479 & -0.3630 & -1.6360 \\
6 & -0.7142 & 0.1354 & 0.0371 \\
& 0.0989 & -0.9355 & 0.0416 \\
& -0.0285 & -0.2247 & -1.2017 \\
7 & -0.6063 & 0.0505 & 0.0130 \\
& 0.0403 & -0.8997 & -0.0889 \\
& -0.0285 & -0.3396 & -1.1767 \\
\hline
\end{tabular}

See Data analysis for a definition of the $X-, Y^{-}$, and $Z$-axes.

$\mathrm{N}(110 \%)$. Force levels were selected in this manner to achieve a comparable level of jaw displacement across subjects despite differences in jaw stiffness.

Pilot tests indicated that when the force pulses were applied along the horizontal perturbation axis, the two higher force magnitudes (90 and $110 \%$ ) consistently exceeded the ability of subjects to compensate. As a result, the three force magnitudes tested in the horizontal direction were 60,70 , and $80 \%$ of the numerical magnitude of jaw stiffness in that direction. Note that under null conditions, jaw stiffness in the horizontal direction was on average 60-90\% higher than stiffness in the vertical and lateral directions.

On average, the three disturbance force magnitudes used in the horizontal direction were $0.89,1.04$, and $1.19 \mathrm{~N}$; the average force magnitudes used in the vertical direction were $0.77,0.91$, and $1.05 \mathrm{~N}$;
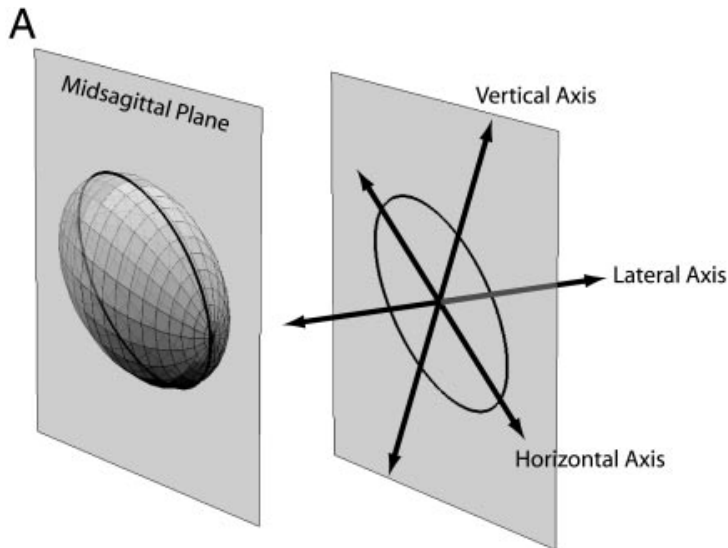

and the average magnitudes used in the lateral direction were 0.57 , 0.70 , and $0.84 \mathrm{~N}$.

We previously demonstrated that the $2 \times 2$ sagittal plane jaw stiffness matrix essentially satisfies the condition of symmetry, indicating that the jaw behaves like a purely springlike system for small-amplitude perturbations (Shiller et al. 2002). In the present study, we have found the same to be true of jaw stiffness in 3D (see RESULTS). This conclusion was based on a decomposition of the jaw stiffness matrix into a symmetric $\left(K_{s}\right)$ and antisymmetric $\left(K_{a}\right)$ component. Subsequent analyses were carried out using only the symmetric component because it alone characterizes the springlike behavior of the system.

A $3 \times 3$ matrix is symmetric if it is equal to its own transpose (i.e., $K=K^{t}$ ). The decomposition of the stiffness matrix $K$ into $K_{s}$ and $K_{a}$ was carried out as follows

$$
\begin{aligned}
& K_{s}=\left[\begin{array}{lll}
K_{x x} & \frac{1}{2}\left(K_{x y}+K_{y x}\right) & \frac{1}{2}\left(K_{x z}+K_{z x}\right) \\
\frac{1}{2}\left(K_{y x}+K_{x y}\right) & K_{y y} & \frac{1}{2}\left(K_{y z}+K_{z y}\right) \\
\frac{1}{2}\left(K_{z x}+K_{x z}\right) & \frac{1}{2}\left(K_{z y}+K_{y z}\right) & K_{z z}
\end{array}\right] \\
& K_{a}=\left[\begin{array}{lll}
0 & \frac{1}{2}\left(K_{x y}-K_{y x}\right) & \frac{1}{2}\left(K_{x z}-K_{z x}\right) \\
\frac{1}{2}\left(K_{y x}-K_{x y}\right) & 0 & \frac{1}{2}\left(K_{y z}-K_{z y}\right) \\
\frac{1}{2}\left(K_{z x}-K_{x z}\right) & \frac{1}{2}\left(K_{z y}-K_{y z}\right. & 0
\end{array}\right]
\end{aligned}
$$

where $K=K_{s}+K_{a}$. Compared with $K_{s}$, the proportion of variance accounted for by $K_{a}$ was found to be negligible (see RESULTS).

The projection of the three principal axes of the jaw stiffness ellipsoid onto the midsagittal plane and its perpendicular (horizontal, vertical, and lateral, shown in Fig. $2 A$ ) were used to characterize the magnitude of jaw stiffness for purposes of comparison among experimental conditions.

\section{RES ULTS}

\section{Stiffness in the null condition}

In the first phase of the experiment, 3D stiffness patterns were estimated in the null condition. Figure $3 A$ shows $3 \mathrm{D}$ stiffness ellipsoids for three subjects, represented in the headcentered coordinate system (in which the occlusal plane defines the horizontal axis). Figure $3 B$ shows the projection of the

B

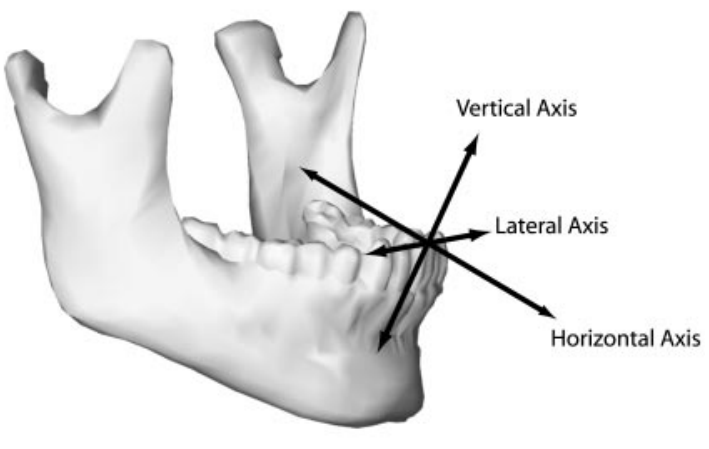

FIG. 2. Disturbance input directions. A: direction of maximum stiffness in the midsagittal plane (horizontal), direction of minimum stiffness in the midsagittal plane (vertical), and a lateral axis orthogonal to the midsagittal plane (lateral). B: same 3 force directions shown relative to the mandible. 
A
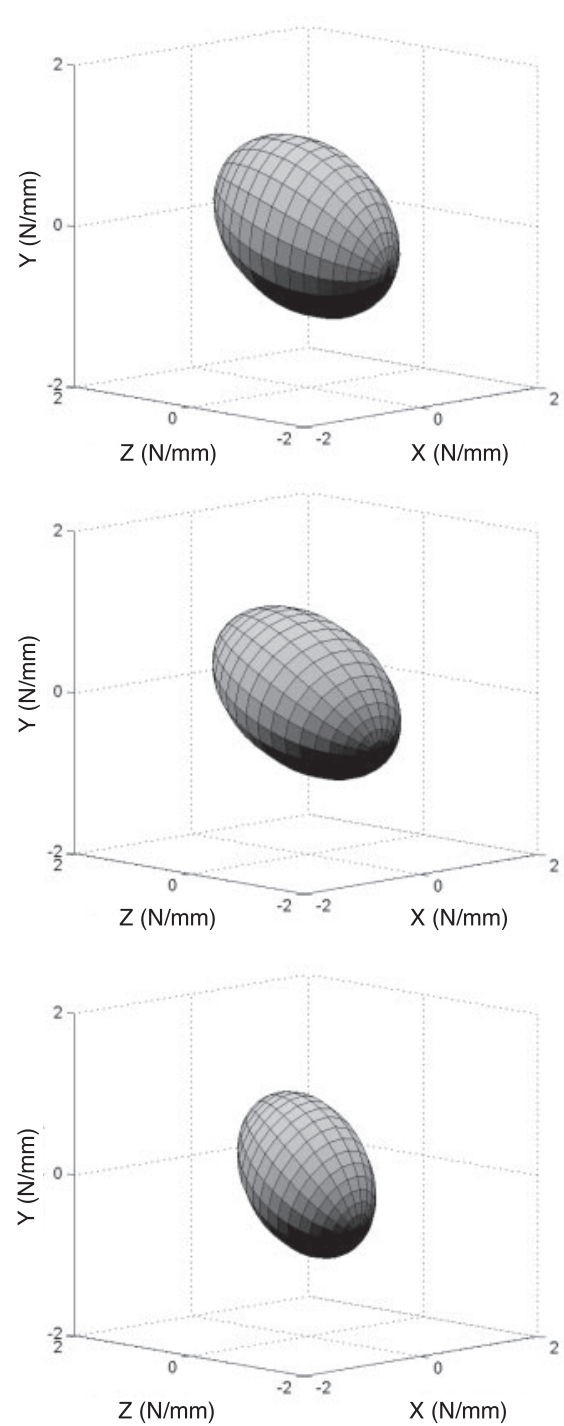

B
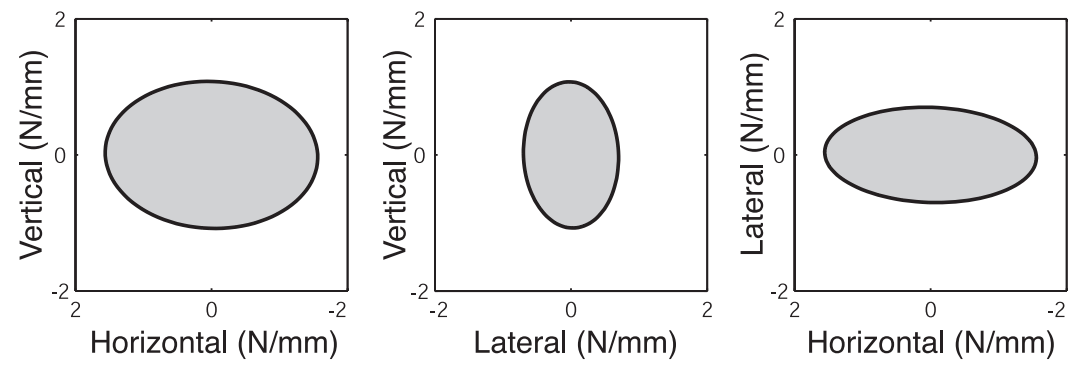

Subject 2
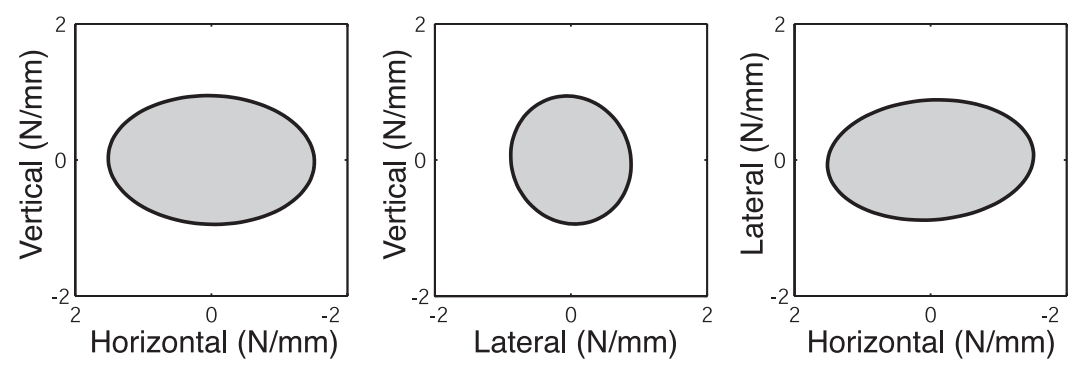

Subject 3
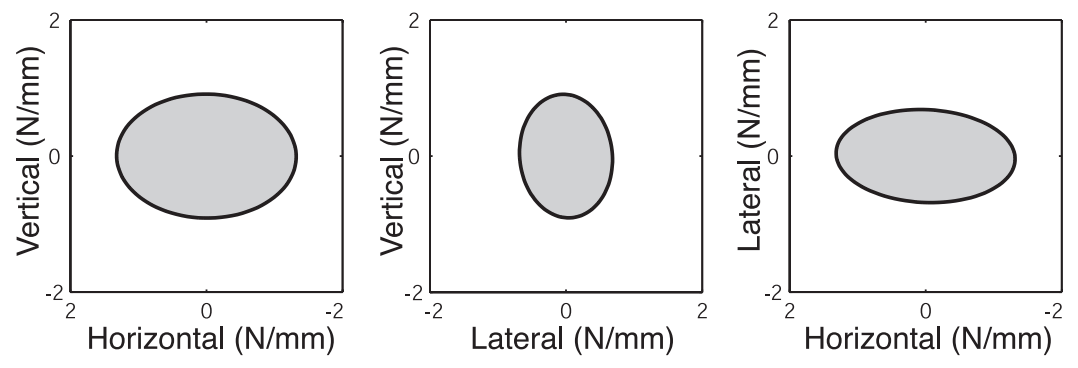

FIG. 3. Stiffness patterns in the null condition. $A$ : stiffness ellipsoids for 3 subjects, represented in the head-centered coordinate system. Recall that the $Z$-axis is aligned with the occlusal plane (the negative direction corresponds to jaw protrusion). $B$ : planar stiffness ellipses represented in coordinates aligned with the 3 axes of the disturbance inputs, shown for the same 3 subjects.

stiffness ellipsoid onto the three planes defined by directions in which disturbances were applied (see METHODS). In general, jaw stiffness patterns were not uniform. The magnitude of stiffness along the horizontal perturbation axis was considerably greater than stiffness on the vertical (jaw raising/lowering) and lateral axes. In addition, stiffness along the vertical axis was greater than stiffness along the lateral axis.

A repeated-measures ANOVA revealed a significant difference in the magnitude of stiffness between the horizontal, vertical, and lateral perturbation axes $(P<0.01)$. Pairwise differences between means were tested using Tukey's method. The stiffness magnitude along the horizontal axis was found to be reliably greater than stiffness on the other two axes $(P<$ 0.01 for both comparisons). Stiffness along the vertical axis was also found to be greater than stiffness along the lateral axis $(P<0.05)$.

Jaw stiffness matrices (representing stiffness in the headcentered coordinate system) are presented for each subject in
Table 1. From each $3 \times 3$ stiffness matrix, an ellipsoid can be produced that characterizes the spatial distribution of jaw stiffness. The magnitude of stiffness corresponding to the three perturbation axes ranged from 1.23 to $1.74 \mathrm{~N} / \mathrm{mm}$ along the horizontal axis (mean $=1.45 \mathrm{~N} / \mathrm{mm}$ ), 0.71 to $1.07 \mathrm{~N} / \mathrm{mm}$ along the vertical axis $($ mean $=0.91 \mathrm{~N} / \mathrm{mm})$, and 0.61 to $0.99 \mathrm{~N} / \mathrm{mm}$ along the lateral axis (mean $=0.76 \mathrm{~N} / \mathrm{mm}$ ). On average, the stiffness ellipsoid was tilted down from the occlusal plane (see Fig. 3A). The mean orientation of the stiffness ellipse in the sagittal plane (measured as the angle between the major axis of the sagittal plane stiffness ellipse and the $Z$-axis) was $153.3^{\circ}$. That is, the ellipsoid was tilted downward from the occlusal plane by $26.7^{\circ}$.

\section{Effect of disturbance force magnitude}

In the second phase of the experiment, jaw stiffness was estimated after the application of force pulses to the jaw. Nine 
A

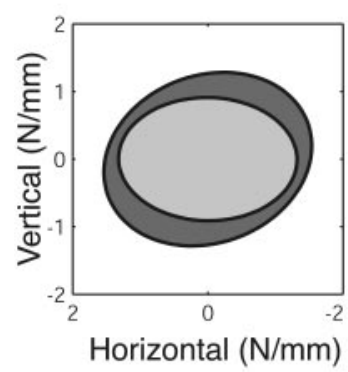

C

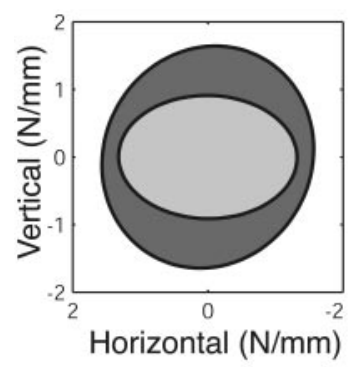

Low Force Level

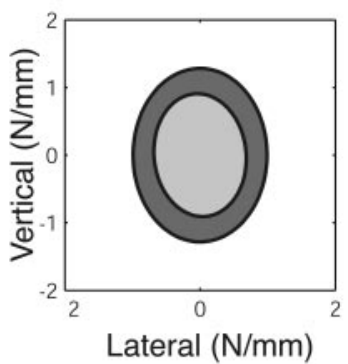

High Force Level

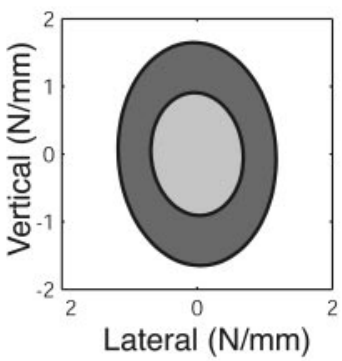

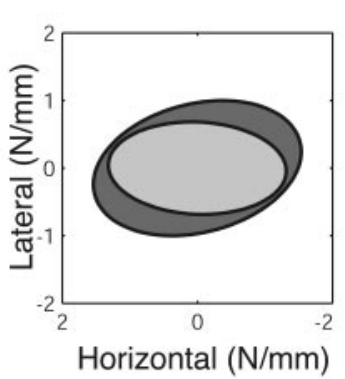

B
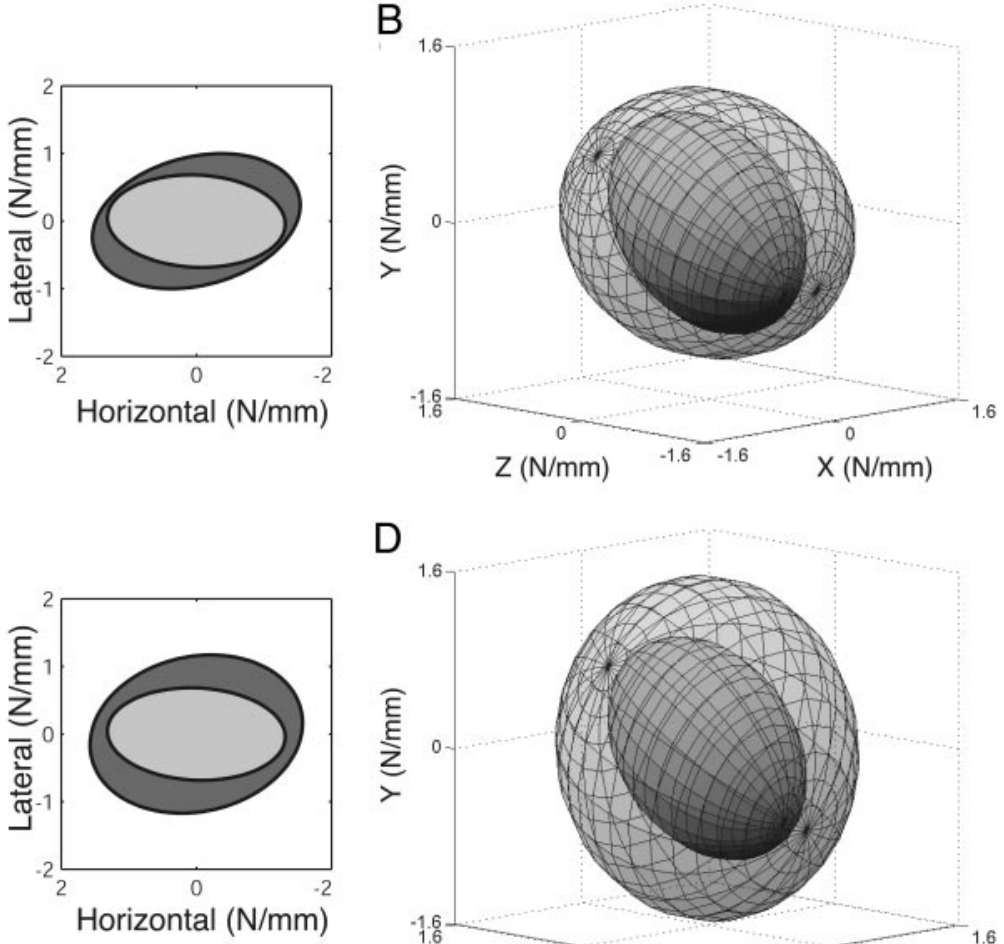

D

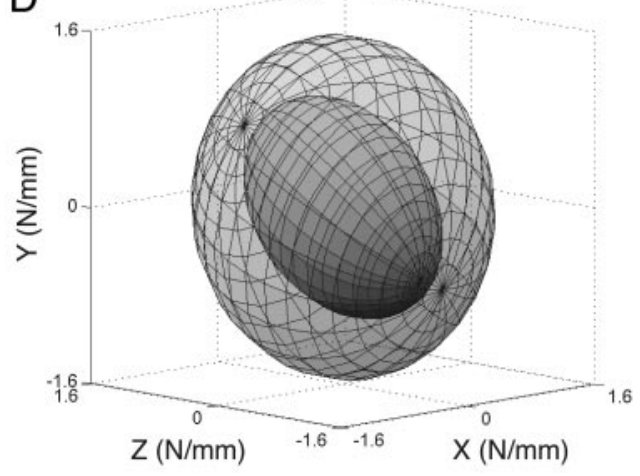

Null Condition With Destabilizing Forces

FIG. 4. Effect of force magnitude on the jaw stiffness ellipse is shown for a single subject. Planar stiffness ellipses (shown to the left) are represented in terms of the 3 perturbation axes. Depiction of the same data as a set of 3D stiffness ellipsoids in head-centered coordinates is shown to the right. Top, A and B: change in stiffness between null conditions (inner ellipses) and the condition involving the lowest level of applied force (outer ellipses). Bottom, $C$ and $D$ : change in stiffness between the null condition and the highest force level. Higher force level is associated with a greater increase in jaw stiffness.

conditions were tested, involving three directions of force application and three force magnitudes. Jaw stiffness patterns were examined in terms of the change in stiffness relative to the stiffness estimates in the null condition.

Figure 4 illustrates the typical effect of the applied force magnitude on the pattern of jaw stiffness for a single subject. In this case, the disturbance acted in the lateral direction. In each panel, the inner ellipse (or ellipsoid) corresponds to stiffness in the null condition and the outer ellipse corresponds to a condition involving the application of force pulses. The top row (Fig. 4, $A$ and $B$ ) shows the change in jaw stiffness associated with the lowest applied force level. The bottom row (Fig. 4, $C$ and $D$ ) shows the change in stiffness for the highest force level. For both magnitudes of disturbance input, an increase in jaw stiffness relative to the null condition can be seen. However, the high force level shows a considerably greater increase in jaw stiffness compared with the low force level.

The effect of the disturbance force magnitude on jaw stiffness across subjects can be seen in Fig. 5. Each panel shows a single direction of force application and the three resulting changes in stiffness at each force level (relative to the null condition). It may be seen that stiffness under conditions of force application was in all cases greater than stiffness in the null condition. In general, greater forces were associated with greater increases in jaw stiffness. This effect was observed for stiffness measured along the vertical and lateral perturbation axes (shown as solid and dashed lines), but not the horizontal axis (dotted line). On average, stiffness along the vertical axis increased relative to the null condition by $39 \%(0.34 \mathrm{~N} / \mathrm{mm})$ for the lowest applied force level (labeled Force Level 1 in Fig. 5 ) and $61 \%(0.52 \mathrm{~N} / \mathrm{mm})$ for the highest force level (Force Level 3). Stiffness along the lateral axis increased by $37 \%$ $(0.27 \mathrm{~N} / \mathrm{mm})$ for the lowest force level and $51 \%(0.37 \mathrm{~N} / \mathrm{mm})$ for the highest level. For the horizontal axis, the stiffness change remained nearly constant, increasing only from 9.2 to $10.2 \%(0.13$ to $0.14 \mathrm{~N} / \mathrm{mm})$ across the three levels of applied force.

Repeated-measures ANOVA was used to examine the effects of applied force magnitude on jaw stiffness using both the proportional and absolute measures of change in stiffness shown in Fig. 5. For both measures, there was a significant effect of applied force level on vertical and lateral jaw stiffness $(P<0.01)$, but not for stiffness along the horizontal axis. However, even for the horizontal axis, stiffness was higher in the presence of disturbance inputs than under null conditions $(P<0.01)$.

\section{Effect of direction of the disturbance input}

When forces were applied in the vertical direction (Fig. 5, middle), the greatest increase in stiffness was observed in that direction (that is, along the vertical axis of the stiffness ellipsoid, shown as a solid line). When forces were applied 
A
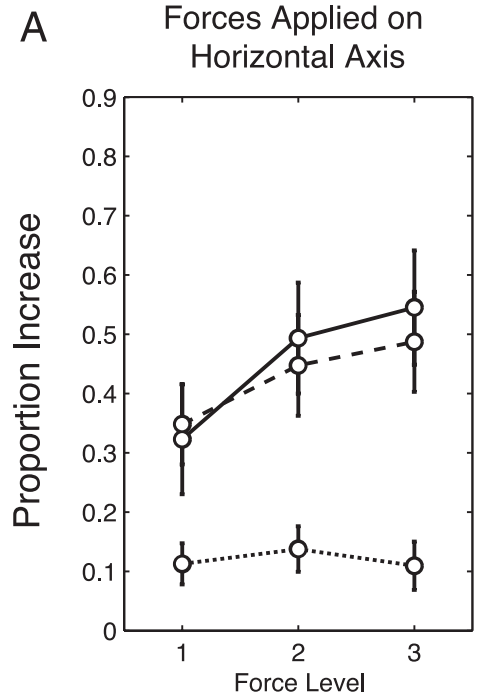

B

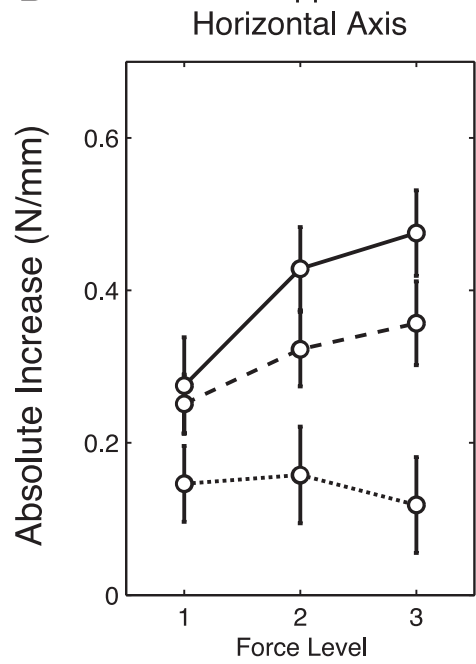

Forces Applied on Vertical Axis

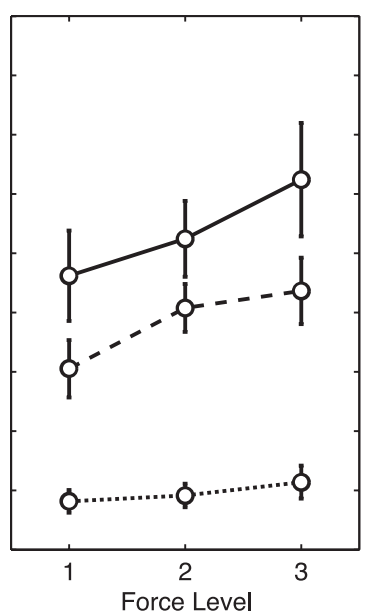

\section{Forces Applied on} Vertical Axis

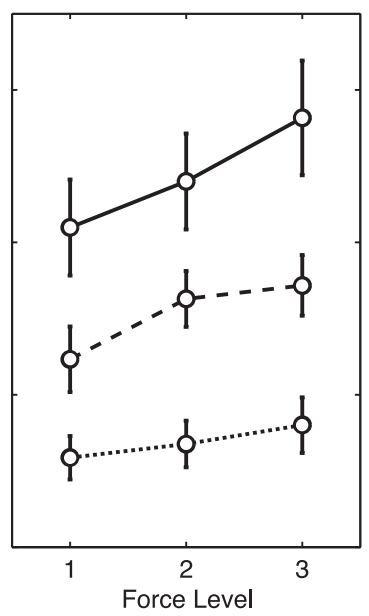

in the horizontal or lateral direction (left and right), the vertical and lateral axes showed a similar degree of stiffness increase. In all cases, stiffness along the horizontal axis (dots) showed no effect of the direction of force application, remaining at about $10 \%$ (or $0.14 \mathrm{~N}$ ) under all conditions.

The details of the changes to stiffness in relation to the direction of the disturbance input are as follows: When forces were applied in the vertical direction, vertical stiffness showed a greater proportional increase than stiffness along the lateral axis $(53.7 \%$ for the vertical axis compared with $38.3 \%$ for the lateral axis). In contrast, stiffness on the vertical and lateral axes increased by nearly the same amount when forces were applied in the horizontal direction (45.4 and $42.8 \%$ increase, respectively) and the lateral direction (51.9 and 53.1\% increase). Similarly, vertical stiffness increased by a greater absolute amount than lateral stiffness when force pulses were applied in the direction of the vertical axis $(0.49 \mathrm{~N} / \mathrm{mm}$ for the vertical axis compared with $0.30 \mathrm{~N} / \mathrm{mm}$ for the lateral axis).

Forces Applied on Lateral Axis

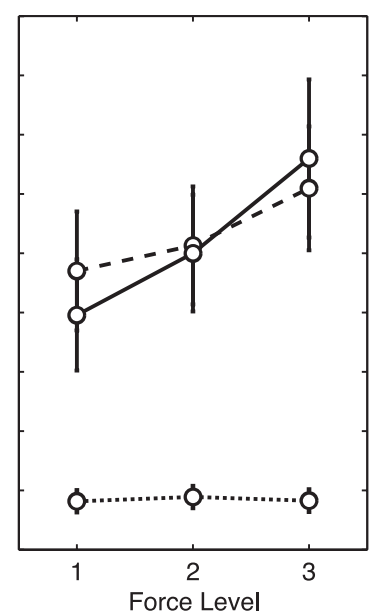

Horizontal Perturbation Axis - Vertical Perturbation Axis

- - Lateral Perturbation Axis

\section{Forces Applied on} Lateral Axis

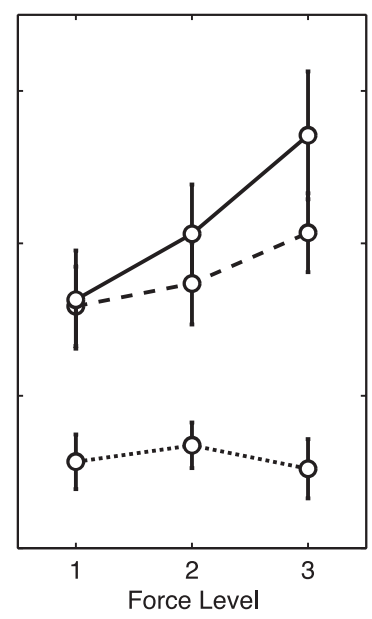

FIG. 5. Mean values of stiffness change in all 9 experimental conditions. Error bars indicate $\pm 1 \mathrm{SE}$. Data are presented as proportional increases in stiffness $(A)$ as well as absolute magnitudes of stiffness change $(B)$. Each of the 3 panels in $A$ and $B$ shows data for a single direction of force application. Three lines in each panel show the change in stiffness separately for the 3 directions of disturbance force application. Three magnitudes of applied forces are shown on the abscissa of each panel (Level 1 is the lowest magnitude; Level 3 is the highest magnitude). Thus for each direction and magnitude of applied force, the resulting change in stiffness in all directions is shown.

When forces were applied in the horizontal and lateral directions, this difference was reduced.

These effects were assessed using Tukey tests of pairwise differences between means. When forces were applied along the vertical axis, the vertical stiffness increase was found to be reliably greater than the increase in the lateral direction, in terms of both proportions $(P<0.05)$ and absolute stiffness change $(P<$ $0.01)$. When forces were applied in the other two directions (horizontal and lateral), no reliable differences between vertical and lateral stiffness change were observed $(P>0.05)$. For all three directions of force application, the change in jaw stiffness along the horizontal axis was significantly less than stiffness change on the other two axes, both in terms of proportional and absolute measures $(P<0.01$ for all comparisons).

\section{Effect of workspace position}

In the present study, jaw stiffness was measured in 3D for a single jaw workspace position (corresponding to the produc- 
tion of the vowel $e$ ). Previous work examining patterns of stiffness in the midsagittal plane revealed a position-dependent effect on the magnitude of jaw stiffness (Shiller et al. 2002). Specifically, for higher jaw positions (closer to occlusion), jaw stiffness was found to be greater (by as much as almost 25\%). We carried out a control study in the absence of load in which we assessed jaw stiffness in 3D at three jaw positions: a high position associated with the production of the vowel $i$ (as in "seed"), a middle position associated with the production of $e$, and a low position associated with the production of $a$ (as in "sad"). Six subjects were tested, using the same procedure for estimating stiffness as described above (null condition). The goal was to determine whether the position-dependent effect on jaw stiffness was present in all directions and, in particular, the lateral axis that was never previously tested.

Figure 6 illustrates the effect of workspace position on jaw stiffness along the horizontal, vertical, and lateral axes. For all three axes, stiffness was found to be greater for the high jaw position compared with the middle and low positions. On average, jaw stiffness at the high position was $10-25 \%$ greater than stiffness at the middle position and 17-35\% higher than stiffness at the low jaw position. Tukey tests revealed significant differences between stiffness at the high and low positions for all three axes $(P<0.01$ for all comparisons). Reliable differences were also observed between the high and middle positions $(P<0.01$ for the horizontal axis, $P<0.05$ for the vertical and lateral axes). No reliable difference was found between the low and middle positions for any of the axes.

\section{Reliability of stiffness estimate}

We have assessed the extent to which the symmetric stiffness matrix captures the empirical pattern of variation in jaw position and restoring force using an $R^{2}$ measure of goodnessof-fit (i.e., the coefficient of determination). The calculation involved the comparison of measured force levels with those predicted by the symmetric stiffness matrix. Specifically

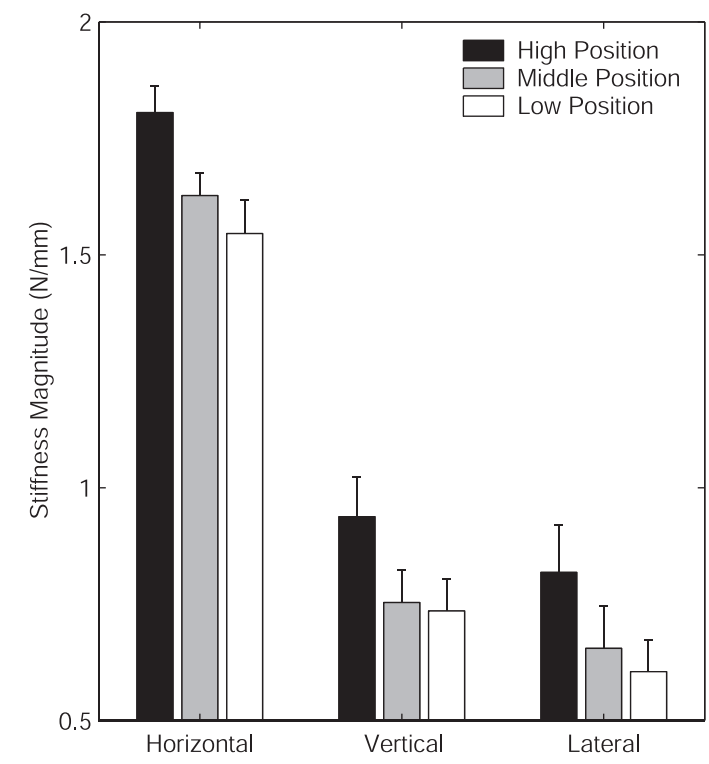

FIG. 6. Effect of jaw workspace position on the magnitude of stiffness along the horizontal, vertical, and lateral axes. Error bars indicate \pm 1 SE. For all 3 axes, stiffness was greater for the high jaw position (closer to occlusion).

$$
R^{2}=1-\frac{\sum(F-\hat{F})^{2}}{\sum(F-\bar{F})^{2}}
$$

where $F$ is the matrix of measured forces, $\hat{F}$ is the matrix of forces predicted by the stiffness matrix, and $\bar{F}$ is the mean of measured forces. The summation $(\Sigma)$ is applied over all three directions of disturbance input. The $R^{2}$ measure was computed separately for each subject and each experimental condition. On average, the proportion of variance accounted for by the symmetric stiffness matrix was $96 \%\left(R^{2}=0.957\right)$, ranging from 93 to $97 \%$ across subjects.

In a related analysis, we found that the three curl components of the $3 \times 3$ stiffness matrix (corresponding to half the difference between corresponding off-diagonal terms; see $E q$. 5 ) were small compared with the diagonal terms of the symmetric stiffness matrix. The mean curl across subjects was $0.061 \mathrm{~N} / \mathrm{mm}$ in the case of estimates of stiffness in the null condition and $0.076 \mathrm{~N} / \mathrm{mm}$ in the case of the higher stiffness values observed in the presence of applied force pulses. On average, in the case of stiffness in the null condition, this corresponds to $4 \%$ of the magnitude of stiffness along the horizontal axis of the stiffness ellipsoid (the axis of maximum stiffness, $1.45 \mathrm{~N} / \mathrm{mm}$ ) and $8 \%$ of the stiffness magnitude along the lateral axis (the axis of minimum stiffness, $0.76 \mathrm{~N} / \mathrm{mm}$ ). Under conditions involving applied forces, the mean magnitude of the curl component corresponds to $5 \%$ of the magnitude of stiffness on the horizontal axis $(1.68 \mathrm{~N} / \mathrm{mm})$ and $7 \%$ of the magnitude of stiffness along the lateral axis $(1.04 \mathrm{~N} / \mathrm{mm})$.

Finally, it was found that the antisymmetric stiffness matrix was able to account for $<1 \%$ of the variance in the empirical data $\left(R^{2}=0.007\right)$. Overall, the relatively small magnitude of the curl component and the extremely limited contribution of the antisymmetric component to the estimate of stiffness indicates that the jaw exhibits primarily springlike behavior in response to small-amplitude perturbations in $3 \mathrm{D}$.

\section{I S C U S S I O N}

In the present study, we have measured the stiffness in the human jaw under conditions of voluntary control. Subjects demonstrated a clear ability to increase the magnitude of jaw stiffness to counteract the effects of a single-axis disturbance. There was a significant increase in stiffness between the null condition and all conditions involving disturbance inputs. The degree to which stiffness was increased was shown to depend on the magnitude of the applied forces, such that greater force magnitudes were associated with greater increases in jaw stiffness. In addition, the manner in which jaw stiffness was adjusted was shown to be dependent on the direction of the disturbance. Specifically, when the disturbance was along a vertical axis, jaw stiffness was found to increase to a greater degree in this direction.

These results support the idea that the magnitude and, in some cases, the spatial distribution of jaw stiffness can be controlled. In studies of arm motion, the control of endpoint stiffness has been shown to play a role in counteracting the effects of unstable interactions with the environment (Burdet et al. 2001; DeSerres and Milner 1991; Lacquaniti et al. 1993) and in the attainment of the differential accuracy requirements of movements (Gribble et al. 2003). The present results suggest that the modulation of stiffness may play a comparable role in the case of orofacial movement. 
The balance of central versus reflex contributions to stiffness merits comment. Because the magnitude of the ramp-and-hold perturbations that were used to measure stiffness was the same under all conditions (i.e., in the null condition and across all levels of disturbance input), the changes that we have observed in stiffness across conditions presumably reflect central change, whether it be to the commands that underlie muscle cocontraction, reflex excitability, or both. Modeling studies suggest that stiffness patterns and stiffness change arise from both central and reflex effects (Gribble et al. 1998). Descending commands that result in changes in the level of muscle cocontraction likewise alter the magnitude of the tonic stretch response. Both are reflected in measures of resistance to displacement.

Stiffness patterns and values are a reflection of jaw geometrical factors, passive tissue properties, active motor units, and reflexes. In the vertical and lateral directions, stiffness was observed to vary in proportion to the magnitude of the disturbance input. In the horizontal direction stiffness change was small and relatively constant in value. This may reflect resistance to displacement that arises in part from the effects of bone and ligament at the condyle. However, the observed effects are not strictly attributed to passive tissue properties; otherwise, no increase in stiffness would have been observed between the null condition and conditions involving the application of disturbing forces (both of which involve the same ramp-and-hold displacements for stiffness measurement). It is worth noting that the measures reported here for stiffness in the horizontal direction are based on displacements of $\leq 1 \mathrm{~mm}$. The functional range of motion of the condyle in the protrusion/retraction direction is in the range of $10 \mathrm{~mm}$. The condyle routinely translates this much in speech production (Ostry and Munhall 1994; Ostry et al. 1997). Thus the displacements applied in the present study are presumably well within the working range.

The ability to modify stiffness globally could serve to reduce variability during speech production and thus play a role in meeting the precision requirements of different speech sounds. In fricative consonants, for example, the tongue must be precisely positioned relative to the palate. Within a given speech sound the requirements for precision may be unequal in different directions. For example, in vowel production, acoustical variation is generally more sensitive to changes in the degree of vocal tract constriction than to the location of the constriction along the vocal tract (Beckman et al. 1995; Gay et al. 1992; Perkell and Nelson 1985; Stevens 1989). The differential precision for different speech sounds or different directions may be achieved at least in part through changes in stiffness.

Stiffness control may also relate more broadly to orofacial function. The ability to counteract the effects of unexpected changes in force acting on the jaw may be necessary for the success of orofacial behaviors such as mastication and speech production. During mastication, the jaw engages in strong mechanical interactions while moving in multiple degrees of freedom. As the food bolus is chewed, its size and mechanical resistance can change dramatically. Under these circumstances, stability is provided by both active and passive tissue properties (Peck et al. 2002) as well as a number of sensory-based mechanisms that are engaged in response to unexpected changes in food resistance. Sudden jaw unloading (resulting, say, from the unexpected breaking of brittle food) and both gradual and rapid increases in resistance (resulting from an encounter with a hard object) are known to evoke short- and long-latency reflex responses in the jaw. Excitatory and inhibitory responses have been documented that are associated with the activity of jaw muscle spindles and periodontal mechanoreceptors (Bjornland et al. 1991; Brodin et al. 1993; Hannam et al. 1970; Lamarre and Lund 1975; Lund et al. 1983; Miles and Wilkinson 1982; Ostry et al. 1997; Poliakov and Miles 1994; Sessle and Schmidt 1972; Türker and Jenkins 2000).

The control of jaw stiffness offers an additional and potentially powerful mechanism for dealing with the effects of sudden changes in load. One particular advantage of stiffness control is that an increase in stiffness arising from muscle cocontraction can precede load changes associated with mechanical interactions and thus provide increased stability with little or no feedback delay (Miles and Wilkinson 1982). Predictive changes to jaw muscle activity and stiffness have been documented in various contexts. Anticipatory changes in muscle activity have been observed before the application of predictable loads to the jaw during cyclic raising and lowering movements (Ottenhoff et al. 1992). Both stiffness change and reflex action in relation to self-generated loads may similarly provide for stability of the jaw in locomotion (Miles et al. 2004; Shiller et al. 2001).

During speech production, the movement of the jaw plays a crucial role in the positioning of the tongue and the lower lip and, through attachments to the hyoid bone, can influence (and be influenced by) laryngeal motion. The rapid motion of any of these structures will generate interaction forces that may affect the position of the jaw and, by extension, any other articulators coupled to it. An increase in jaw stiffness would limit the influence of any loads to the jaw arising from the motion of other articulators, thereby reducing the possibly disruptive effects of forces associated with mechanically coupled articulator motions. The ability to scale the magnitude of stiffness to match precisely the effect of externally applied forces, and the ability to alter stiffness differentially in directions relevant to speech production, may thus facilitate the control of speech movements.

It is important to consider the relationship between stiffness and muscle force generation when examining the voluntary control of stiffness. It is well known that increases in joint stiffness accompany the production of active joint torques (Hunter and Kearney 1982; Weiss et al. 1986, 1988). Such changes are a natural consequence of reflexes and muscle mechanical properties-specifically, the relation between a muscle's resistance to stretch and its level of activation-and do not necessarily reflect the control of stiffness per se by the nervous system (Hoffer and Andreassen 1981; Rack and Westbury 1969). The coupling between stiffness and force production leads to a difficulty in interpreting results such as those by Cooker and colleagues (1980) in which they reported measures of jaw stiffness during the production of a sustained $10-\mathrm{N}$ bite force. The magnitude of stiffness observed under such conditions was high (ranging from 10 to $15 \mathrm{~N} / \mathrm{mm}$ ) compared with measures of stiffness under conditions involving no net force production (Shiller et al. 2002). On the basis of EMG measures, Cooker and colleagues concluded that the stretch reflex was a major contributor to the jaw's springlike behavior. However, it remains unknown whether the difference in stiff- 
ness between conditions involving applied bite force and conditions involving no external loads is attributable simply to the increase in jaw closer muscle activity associated with biteforce production.

In light of such issues, a major challenge in studies of stiffness control is to distinguish the changes in stiffness that naturally accompany changes in joint torque from the ability to modify stiffness when the net torque is zero and the limb or articulator is stationary. Although both types of stiffness change may be functionally significant, the ability to control stiffness independently of force production is a potentially powerful mechanism for maintaining the positional stability of the motor system. In particular, it would allow for the adjustment of stiffness before any loads are applied to the system, in anticipation of an unstable or unpredictable mechanical interaction. In the present study, different magnitudes of force pulses were applied to elicit an increase in jaw stiffness, although the actual measurement of stiffness occurred under static conditions in which no external forces were present. Because the jaw was stationary, the observed changes in stiffness were not associated with any changes in net force applied by the jaw. Thus one can conclude that subjects were in fact controlling jaw stiffness.

In addition to demonstrating the voluntarily control of jaw stiffness, the present study provides the first description of jaw stiffness in 3D. Jaw stiffness in the midsagittal plane has been investigated previously (Shiller et al. 2002), revealing a nonuniform spatial distribution with stiffness being greatest along a roughly horizontal axis (in the direction of jaw protrusion and retraction) and least in the direction of jaw raising and lowering. The present results reveal that jaw stiffness in the lateral degree of freedom (orthogonal to the sagittal plane) is less than stiffness in the vertical direction by about $15 \%$ (or $0.25 \mathrm{~N} / \mathrm{mm}$ ). This is a considerably smaller difference than the nearly $60 \%$ $(0.59 \mathrm{~N} / \mathrm{mm})$ difference between the vertical and horizontal axes of the midsagittal stiffness ellipse. Therefore compared with the highly anisotropic pattern of stiffness in the midsagittal plane, the frontal-plane stiffness pattern (consisting of the vertical and lateral axes) is more uniform.

\section{G R A N T S}

This research was supported by National Institute on Deafness and Other Communication Disorders Grant DC-04669, National Sciences and Engineering Research Council, Canada, and Fonds Québécois de la Recherche sur la Nature et les Technologies, Quebec.

\section{REFERENCES}

Beckman ME, Jung T, Lee S, de Jong K, Krishnamurthy AK, Ahalt SC, Cohen KB, and Collins MJ. Variability in the production of quantal vowels revisited. J Acoust Soc Am 97: 471-490, 1995.

Bjornland T, Brodin P, and Aars H. Force-related changes in the masseter muscle reflex response to tooth-taps in man. J Oral Rehabil 18: 125-132, 1991.

Brodin P, Türker KS, and Miles TS. Mechanoreceptors around the tooth evoke inhibitory and excitatory reflexes in the human masseter muscle. J Physiol 464: 711-723, 1993.

Burdet E, Osu R, Franklin DW, Milner TE, and Kawato M. The central nervous system stabilizes unstable dynamics by learning optimal impedance. Nature 414: 446-449, 2001.

Cooker HS, Larson CR, and Luschei ES. Evidence that the human jaw stretch reflex increases the resistance of the mandible to small displacements. J Physiol 308: 61-78, 1980.

Darainy M, Malfait N, Gribble PL, Towhidkhah F, and Ostry DJ. Learning to control arm stiffness under static conditions. J Neurophysiol 92: 3344-3350, 2004.
DeSerres SJ and Milner TE. Wrist muscle activation patterns and stiffness associated with stable and unstable mechanical loads. Exp Brain Res 86: 451-458, 1991.

Flash T and Mussa-Ivaldi FA. Human arm characteristics during the maintenance of posture. Exp Brain Res 82: 315-326, 1990.

Franklin DW, Osu R, Burdet E, Kawato M, and Milner TE. Adaptation to stable and unstable dynamics achieved by combined impedance control and inverse dynamics model. J Neurophysiol 90: 3270-3282, 2003.

Gay T, Boë LJ, and Perrier P. Acoustic and perceptual effects of changes in vocal tract constrictions for vowels. J Acoust Soc Am 92: 1301-1309, 1992.

Gomi H and Osu R. Task-dependent viscoelasticity of human multijoint arm and its spatial characteristics for interaction with environments. $J$ Neurosci 18: 8965-8978, 1998.

Gribble PL, Mullin LI, Cothros N, and Mattar A. Role of cocontraction in arm movement accuracy. J Neurophysiol 89: 2396-2405, 2003.

Gribble PL, Ostry DJ, Sanguineti V, and Laboissière R. Are complex control signals required for human arm movement? J Neurophysiol 79: 1409-1424, 1998.

Guenther FH, Espy-Wilson CY, Boyce SE, Matthies ML, Zandipour M, and Perkell JS. Articulatory tradeoffs reduce acoustic variability during American English /r/ production. J Acoust Soc Am 105: 2854-2865, 1999.

Hannam AG, Matthews B, and Yemm R. Receptors involved in the response of the masseter muscle to tooth contact in man. Arch Oral Biol 15: 17-24, 1970.

Hoffer JA and Andreassen S. Regulation of soleus muscle stiffness in premammillary cats: intrinsic and reflex components. J Neurophysiol 45: $267-285,1981$.

Hogan N. The mechanics of multi-joint posture and movement control. Biol Cybern 52: 315-351, 1985.

Hunter IW and Kearney RE. Dynamics of human ankle stiffness: variation with mean ankle torque. J Biomech 15: 747-752, 1982.

Lacquaniti F, Carrozzo M, and Borghese NA. Time-varying mechanical behavior of multijointed arm in man. J Neurophysiol 69: 1443-1464, 1993.

Lamarre $\mathbf{Y}$ and Lund JP. Load compensation in human masseter muscles. J Physiol 253: 21-35, 1975.

Lund JP, Lamarre Y, Lavigne G, and Duquet G. Human jaw reflexes. Adv Neurol 39: 739-755, 1983.

McIntyre J, Mussa-Ivaldi FA, and Bizzi E. The control of stable postures in the multijoint arm. Exp Brain Res 110: 248-264, 1996.

Miles TS, Flavel SC, and Nordstrom MA. Control of human mandibular posture during locomotion. J Physiol 554: 216-226, 2004.

Miles TS and Wilkinson TM. Limitation of jaw movement by antagonist muscle stiffness during unloading of human jaw closing musclces. Exp Brain Res 46: 305-310, 1982.

Milner TE. Adaptation to destabilizing dynamics by means of muscle cocontraction. Exp Brain Res 143: 406-416, 2002.

Milner TE and Cloutier C. Compensation for mechanically unstable loading in voluntary wrist movement. Exp Brain Res 94: 522-532, 1993.

Milner TE, Cloutier C, Leger AB, and Franklin DW. Inability to activate muscles maximally during cocontraction and the effect on joint stiffness. Exp Brain Res 107: 293-305, 1995.

Mussa-Ivaldi FA, Hogan N, and Bizzi E. Neural, mechanical, and geometric factors subserving arm posture in humans. J Neurosci 5: 2732-2743, 1985.

Ostry DJ, Gribble PL, Levin M, and Feldman AG. Phasic and tonic stretch reflexes in muscles with few muscle spindles: human jaw opener muscles. Exp Brain Res 116: 299-308, 1997.

Ostry DJ and Munhall KG. Control of jaw orientation and position in mastication and speech. J Neurophysiol 71: 1528-1545, 1994.

Ostry DJ, Vatikiotis-Bateson E, and Gribble PL. An examination of the degrees of freedom of human jaw motion in speech and mastication. $J$ Speech Lang Hear Res 40: 1341-1351, 1997.

Ottenhoff FAM, van der Bilt A, van der Glas HW, and Bosman F. Peripherally induced and anticipating elevator muscle activity during simulated chewing in humans. J Neurophysiol 67: 75-83, 1992.

Peck CC, Sooch AS, and Hannam AG. Forces resisting jaw displacement in relaxed humans: a predominantly viscous phenomenon. J Oral Rehab 29: 151-160, 2002.

Perkell JS, Matthies ML, Svirsky MA, and Jordan MI. Trading relations between tongue-body raising and lip rounding in production of the vowel /u/: a pilot "motor equivalence" study. J Acoust Soc Am 93: 2948-2961, 1993.

Perkell JS and Nelson WL. Variability in production of the vowels /i/ and /a/. J Acoust Soc Am 77: 1889-1895, 1985.

Perreault EJ, Kirsch RF, and Crago PE. Voluntary control of static endpoint stiffness during force regulation tasks. J Neurophysiol 87: 2808-2816, 2002. 
Poliakov AV and Miles TS. Stretch reflexes in human masseter. $J$ Physiol 476: 323-331, 1994.

Rack PM and Westbury DR. The effects of length and stimulus rate on tension in the isometric cat soleus muscle. J Physiol 204: 443-460, 1969.

Savariaux C, Perrier P, and Orliaguet JP. Compensation strategies for the perturbation of the rounded vowel /u/ using a lip tube: a study of the control space in speech production. J Acoust Soc Am 98: 2428-2442, 1995.

Sessle BJ and Schmitt A. Effects of controlled tooth stimulation on jaw muscle activity in man. Arch Oral Biol 17: 1597-1607, 1972.

Shiller DM, Ostry DJ, Gribble PL, and Laboissière R. Compensation for the effects of head acceleration on jaw movement in speech. J Neurosci 21 : 6447-6456, 2001.
Shiller DM, Ostry DJ, and Laboissière R. The relationship between jaw stiffness and kinematic variability in speech. J Neurophysiol 88: 2329 2340, 2002.

Stevens KN. On the quantal nature of speech. J Phon 17: 3-45, 1989.

Türker KS, and Jenkins M. Reflex responses induced by tooth unloading. J Neurophysiol 84: 1088-1092, 2000.

Weiss PL, Hunter IW, and Kearney RE. Human ankle joint stiffness over the full range of muscle activation levels. J Biomech 21: 539-544, 1988.

Weiss PL, Kearney RE, and Hunter IW. Position dependence of ankle joint dynamics-ii. Active mechanics. J Biomech 19: 737-751, 1986. 